\title{
Effect of Class IV Laser Therapy on Chemotherapy-Induced Oral Mucositis
}

\section{A Clinical and Experimental Study}

\author{
Giulia Ottaviani, ${ }^{*}$ Margherita Gobbo, ${ }^{*}$ Mauro Sturnega ${ }^{\dagger}$ Valentina Martinelli, ${ }^{\dagger}$ Miguel Mano, ${ }^{\dagger}$ Fabrizio Zanconati, ${ }^{\dagger}$ \\ Rossana Bussani, ${ }^{\ddagger}$ Giuseppe Perinetti, ${ }^{*}$ Carlin S. Long, ${ }^{\S}$ Roberto Di Lenarda, ${ }^{*}$ Mauro Giacca, ${ }^{\dagger}$ Matteo Biasotto, ${ }^{*}$ and \\ Serena Zacchigna
}

From the Division of Oral Pathology, ${ }^{*}$ Dental Science Department, and the Department of Histology and Pathology, ${ }^{\ddagger}$ University of Trieste, Trieste, Italy; the Molecular Medicine Laboratory, ${ }^{\dagger}$ International Centre for Genetic Engineering and Biotechnology (ICGEB), Trieste, Italy; and the University of Colorado Health Sciences Center, ${ }^{\S}$ Denver, Colorado

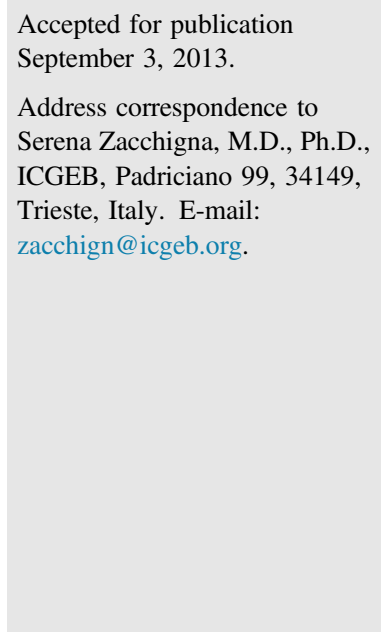

\begin{abstract}
Oral mucositis $(0 M)$ is a serious and acute side effect in patients with cancer who receive chemotherapy or radiotherapy, often leading to the suspension of therapy and a need for opioid analgesic and enteral/ parenteral nutrition, with an effect on patient survival. Among the various interventions proposed in OM management, laser therapy is becoming a recommended treatment option but has limitations due to its heterogeneous laser parameters. Here, we report on our successful clinical experience on the use of class IV laser therapy to treat OM induced by different chemotherapy regimens. To shed light on the mechanisms of action of laser therapy in improving $0 M$ resolution, we have developed an animal model of chemotherapy-induced $0 \mathrm{M}$, in which we compare the efficacy of the standard low-power laser therapy protocol with an innovative protocol, defined as high-power laser therapy. We show that high-power laser therapy is more effective than low-power laser therapy in improving 0M lesion healing, reducing the inflammatory burden, and preserving tissue integrity. In addition, high-power laser therapy has been particularly effective in promoting the formation of new arterioles within the granulation tissue. Our results provide important insights into the mechanism of action of biostimulating laser therapy on $\mathrm{OM}$ in vivo and pave a way for clinical experimentation with the use of high-power laser therapy. (Am J Pathol 2013, 183: 1747-1757; http://dx.doi.org/10.1016/j.ajpath.2013.09.003)
\end{abstract}

Mounting evidence indicates that more aggressive regimens of radiotherapy (RT) and/or chemotherapy (CT) improve loco-regional control and overall survival in patients with cancer. Better treatment outcome, however, often comes at the expense of increased patient morbidity in terms of debilitating side effects, such as nausea, vomiting, diarrhea, and mucositis. In particular oral mucositis (OM) is the inflammation of the mucous membrane of the oral and oropharyngeal region and is referred to as one of the main problems of patients with cancer who receive head and neck RT, hematopoietic stem cell transplantation, and myelosuppressive CT for solid tumors. ${ }^{1}$ This condition results in a continuum of clinical changes that range from erythema and burning of the oral and oropharyngeal mucosa to the development of diffuse and confluent ulcerations. ${ }^{2}$ The percentage of patients experiencing OM as a consequence of CT lies between $5 \%$ and $45 \%$, but rises to between $70 \%$ and $100 \%$ when considering high-dose CT and/or association with hematopoietic stem cell transplantation. ${ }^{2} \mathrm{OM}$ is extremely debilitating because it affects swallowing, chewing, and phonation and, hence, significantly worsens quality of life. $^{3}$ Disparity exists between perceived symptoms and objective severity of the lesions, which, in some instances, lead to life-threatening complications, such as hemorrhage,

Supported by grants from the Fondazione CR Trieste, Trieste, Italy (M.G.) and from the Associazione Italiana per la Ricerca sul Cancro (AIRC) (S.Z.). 
infection, or tracheotomy, thereby imposing reduction or suspension of $\mathrm{CT}$ and worsening of the underlying neoplastic disease. Patients affected by OM need prolonged morphine and parenteral nutrition, resulting in prolonged hospitalization and subsequent economic burden. ${ }^{4}$

Few treatment options for OM are approved, and these options are essentially limited to minimally effective preventive and palliative remedies. During the past 20 years, many therapeutic agents have been proposed in the clinical management of OM. These therapeutic agents have included azelastine, glutamine, sucralfate, granulocyte macrophage colony stimulating factor, granulocyte colony stimulating factor, transforming growth factor $\beta 3$, IL-11, fibroblast growth factors, keratinocyte growth factor, reactive oxygen species inhibitors (amifostine, $N$ acetylcysteine), sulfate morphine, pilocarpine, pentoxiphylline, prednisone, benzydamine, allopurinol, chlorexidine, and hydrogels. ${ }^{5-8}$ In all these cases, however, the lesions often become refractory to any treatment, necessitating the use of topical and systemic opioid analgesics. Outcomes of the current treatments are controversial, and a standard therapy is not yet available. Recent findings indicate that biostimulating laser therapy can usefully contribute to OM management. In particular, the local application of a monochromatic, narrow-band, coherent light source (600 to $1000 \mathrm{~nm}$ ) represents an effective approach to improve wound and soft tissue healing, reduce inflammation, and provide relief for both acute and chronic pain. The mechanism of laser biostimulation is disputed, but a cytoprotective effect against oxidative stress has been observed in various cellular models. ${ }^{9}$ The capacity of the wavelengths used to penetrate tissues, in all likelihood, allows for the direct activation of intracellular chromophores, resulting in increased ATP production by the mitochondrial respiratory chain, ${ }^{10}$ as well as in increased proliferation of several cell types, including endothelial cells, keratinocytes, fibroblasts, osteoblasts, and pericytes, ${ }^{11-13}$ resulting in the prompt formation and activity of granulation tissue. In addition, some support exists for the capacity of laser to stimulate vascular smooth muscle relaxation, thus contributing to the analgesic effects of laser therapy. ${ }^{11}$ On the basis of these considerations, biostimulating laser therapy has been proposed as an innovative tool to manage CT-induced OM, although agreement is not uniform on parameters such as wavelength, power, and duration of treatment. ${ }^{14}$

Lasers are classified by their ability to cause biological damage. Biostimulating protocols usually exploit class I, II, and III lasers, which work at $1-\mathrm{W}$ power or less and are therefore conventionally defined as low-level laser therapy. Different from the traditional approach, here we report on our clinical experience about the efficacy of a class IV laser device on OM induced by CT. The most recent classification of biostimulating lasers, as well as of the protocols used in our study, is reported in Supplemental Table S1. In addition, we show the results of an experimental study that was based on a mouse model of CT-induced OM, in which we have compared the effect of the clinical protocol (herein
Table 1 Selected Baseline Characteristics of Patients with CTInduced $0 \mathrm{M}$

\begin{tabular}{lccl}
\hline Characteristics & $\begin{array}{l}\text { Hematologic } \\
\text { cancer }\end{array}$ & $\begin{array}{l}\text { Head and } \\
\text { neck cancer }\end{array}$ & $\begin{array}{l}\text { Solid cancer } \\
\text { (other districts) }\end{array}$ \\
\hline No & 6 & 2 & 12 \\
Sex & & & \\
$\quad$ Female & 5 & 1 & 5 \\
$\quad$ Male & 1 & 1 & 7 \\
Mean age (years) & 50.5 & 72.5 & 67.6 \\
VAS (mean at T0) & 5.8 & 4.5 & 4.3 \\
CTC (mean at T0) & 2.3 & 3.5 & 2.1 \\
\hline
\end{tabular}

referred as LPLT, low-power laser therapy) with an innovative protocol that was based on a higher wavelength and energy power (herein referred as HPLT, high-power laser therapy), which has been used, recently and successfully, in the treatment of patients affected by cetuximab-associated acneiform rash. ${ }^{15}$

\section{Materials and Methods}

\section{Patient Population}

Eligible participants were 20 to 85 years old and were affected by CT-related OM, due to three classes of neoplastic diseases: hematological malignancies (class 1), head and neck cancers (class 2), and solid tumors (class 3) (Table 1). Exclusions from participation included ongoing or previous RT on head and/or neck and inability to adhere to the whole duration of the protocol. Participants were recruited from patients attending the Oral Medicine and Pathology Department, Ospedale Maggiore, Trieste, Italy.

\section{Clinical Protocol}

Between March 2010 and January 2012, 20 eligible patients ( 9 men and 11 women) were enrolled in the study. All patients were enrolled according to protocols approved by the Ethical Committee of the Azienda Ospedaliero-Universitaria Ospedali Riuniti di Trieste, after written informed consent was obtained. A scheme of the study design is shown in Figure 1A.

During a first visit (day 1), patients were subjected to a detailed questionnaire to collect information about their clinical history and to evaluate OM severity. A visual analog scale (VAS) was used to evaluate the following subjective parameters: i) voice alteration; ii) swallowing difficulties; iii) lips, saliva, tongue, and mucosa alterations; and iv) difficulty in performing a correct oral hygiene. The oral cavity was then examined to classify $\mathrm{OM}$ and to characterize ulceration and erythema distribution and size, according to the common toxicity criteria (CTC) scale ${ }^{16}$ (Table 2). The following nine areas were considered: upper and lower lips, right and left cheeks, right and left sides of tongue, floor of mouth, and soft and hard palates (Figure 2A). 


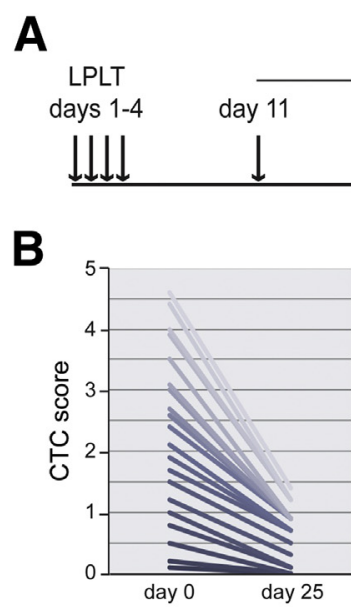

Follow-up

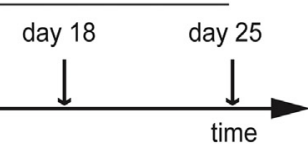

D
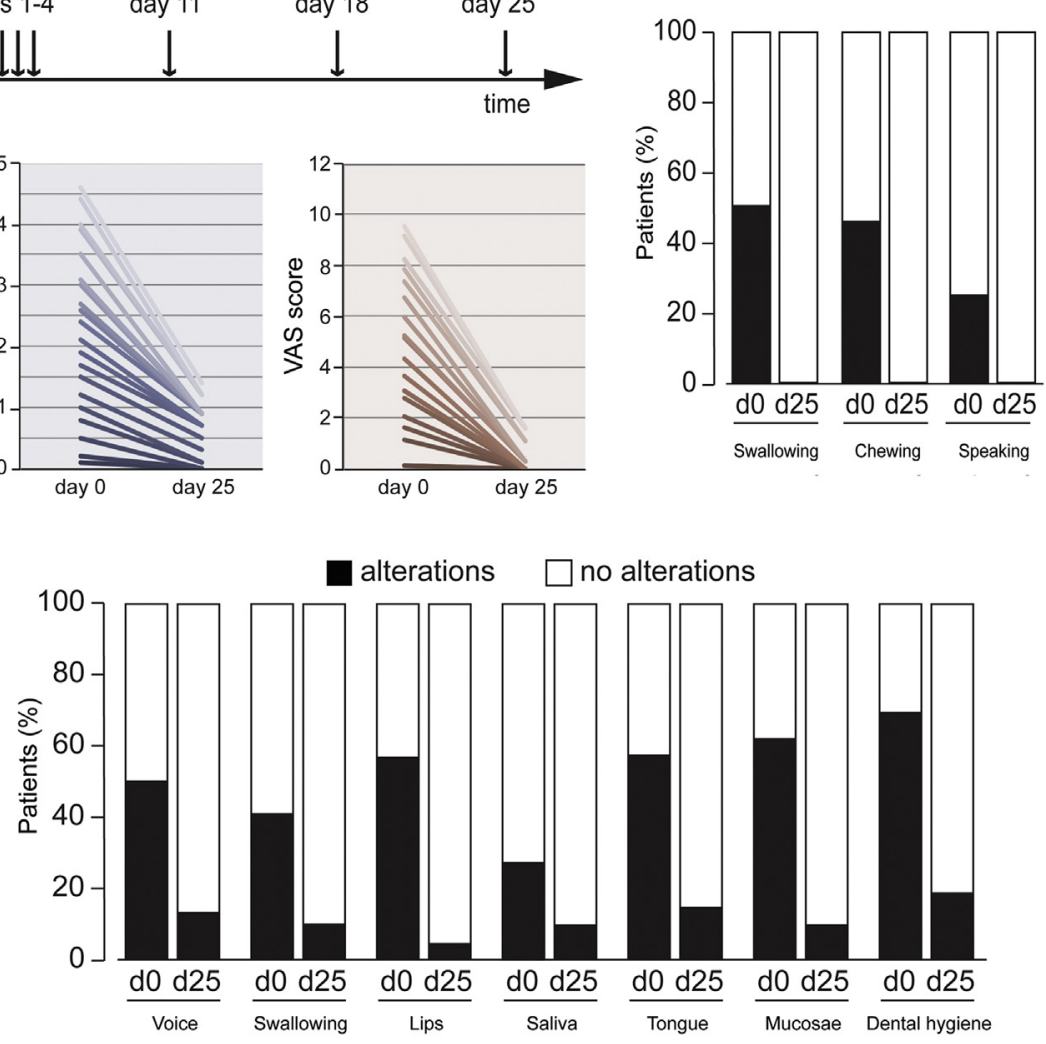

Figure 1 Effect of LPLT on chemotherapyinduced OM. A: Schematic representation of the study design. Enrolled patients were treated with LPLT for 4 consecutive days, followed by a 3-week follow-up period. B: Evaluation of OM severity by CTC scale (left panel) and by a VAS (right panel) at days 0 and 25. A line joins the scores attributed to the same patient at the two time points, to underscore the decreasing trend in OM severity in all of the patients. C: Indication of the number of patients indicating presence (black bars) or absence (white bars) of pain at swallowing, chewing, or speaking at day 0 (d0) and day 25 (d25). D: Indication of the number of patients indicating subjective alterations of the following parameters at day 0 (d0) and day 25 (d25): voice, swallowing capacity, lip integrity, salivation, tongue integrity, oral mucosa integrity, and ability to perform a proper dental hygiene.
On days 1 to 4 , all patients were subjected to laser sessions, using a diode laser (class IV, series number 00027; Eltech S.r.1, Treviso, Italy) and the following parameters: wavelength $635 \mathrm{~nm}$, frequency $2 \mathrm{~Hz}$, power $2.5 \mathrm{~mW}$, time 180 seconds, spot size $1 \mathrm{~cm}^{2}$, and total energy $0.45 \mathrm{~J}$ in defocused mode. Before laser therapy, all patients were subjected to a protocol of oral hygiene, which was periodically repeated to standardize conditions. Every day, laser application was repeated twice, with a 5-minute interval and followed by a simplified questionnaire to evaluate swallowing, chewing, and phonatory pain, according to VAS and CTC. Both the patient and the operator used protective glasses to prevent the risk of eye damage. Laser pointer was taken 1 to $3 \mathrm{~cm}$ far from the affected areas, and a rotator motion was applied. During the 3-week follow-up, every patient was seen once a week (day 11, 18, and 25), and the OM status was evaluated by both VAS and CTC.

\section{Mouse Model of OM}

Animal care and treatment were conducted in conformity with institutional guidelines in compliance with national and international laws and policies (European Economic Community Council Directive 86/609, OJL 358, December 12, 1987).

A total of $48 \mathrm{C} 57 \mathrm{BL} / 6$ female mice with a mean weight of $18 \mathrm{~g}, 6$ to 7 weeks old, were used for this study and housed under controlled environmental conditions for 5 days.

OM was induced in all animals with two doses of 5fluorouracil at $60 \mathrm{mg} / \mathrm{kg}$ on days -4 and -2 , respectively. On day 0, after general anesthesia with $100 \mathrm{~mL} / 10 \mathrm{mg}$ Avertin 2\%, a 4-mm square of Wattman 3-mm thick chromatography paper soaked in $50 \%$ acetic acid was applied to the underside of the tongue for 60 seconds, as a mucosal irritant to accelerate the induction process.

Animals were randomly assigned into the following three groups: control group of 16 mice in which no laser therapy was applied; the LPLT group of 16 mice treated with LPLT protocol $(\lambda 635 \mathrm{~nm}, 2.5 \mathrm{~mW}$ in continuous wave, $2 \mathrm{~Hz}, 30 \mathrm{sec}-$ onds, spot size diameter $0.5 \mathrm{~cm}$, energy density $0.375 \mathrm{~J} / \mathrm{cm}^{2}$, $5 \mathrm{~cm}$ distance), and the HPLT group of 16 mice treated with HPLT protocol ( $\lambda 970 \mathrm{~nm}, 5 \mathrm{~W}$, duty cycle 50\%, $2 \mathrm{~Hz}, 30$ seconds, spot size diameter $0.5 \mathrm{~cm}$, energy density $375 \mathrm{~J} / \mathrm{cm}^{2}$, $5 \mathrm{~cm}$ distance).

Similar to the clinical condition, laser therapy was applied for 4 consecutive days (day 1 to 4 ), keeping the laser pointer $1 \mathrm{~cm}$ from the tongue and being continuously moved during therapy.

During days 1 to 10 , animals were monitored individually for survival, body weight, and assessment of OM, according to a severity score displayed in Supplemental Table S2. Excisional biopsies were obtained from the tongue of all 
Table 2 CTC scale

\begin{tabular}{|c|c|c|c|c|}
\hline Grade 0 & Grade 1 & Grade 2 & Grade 3 & Grade 4 \\
\hline None & $\begin{array}{l}\text { Erythema of the mucosa } \\
\text { Asymptomatic or mild } \\
\text { symptoms; normal diet }\end{array}$ & $\begin{array}{l}\text { Patchy ulcerations; } \\
\text { pseudomembranes } \\
\text { Moderate pain; not interfering } \\
\text { with oral intake; modified } \\
\text { diet indicated }\end{array}$ & $\begin{array}{l}\text { Confluent ulcerations or } \\
\text { pseudomembrane; bleeding } \\
\text { with minor trauma } \\
\text { Severe pain, interfering } \\
\text { with oral intake }\end{array}$ & $\begin{array}{l}\text { Tissue necrosis; significant } \\
\text { spontaneous bleeding; } \\
\text { life-threatening consequences }\end{array}$ \\
\hline
\end{tabular}

animals according to the schedule shown in Supplemental Table S3. Biopsy specimens were fixed in $4 \%$ formalin and embedded in paraffin for histological analysis.

$\alpha$-SMA-RFP/Coll $\alpha 1(\mathrm{I})-\mathrm{EGFP}$ mice, ${ }^{17}$ which simultaneously express the red fluorescent protein (RFP) under the control of the $\alpha$-smooth muscle actin ( $\alpha$-SMA) promoter and the enhanced green fluorescent protein (EGFP) under the control of the collagen $\alpha 1$ (I) promoter, were the kind gift of David Brenner to Carlin S. Long. These mice were exposed to the experimental procedures as were the C57BL/6 mice.

\section{Cell Culture and Irradiation}

Human coronary artery smooth muscle cells (SMCs) were cultured in SmcGM culture medium (both cells and medium were purchased from Lonza, Basel, Switzerland) and were irradiated with the same diode laser used for clinical and animal studies, but equipped with an adapted probe specifically designed to provide a uniform irradiation to a 96-multiwell plate (a prototype was provided by Eltech S.r.l). Cultured cells were exposed to either HPLT $(\lambda 970 \mathrm{~nm}, 20 \mathrm{~mW}$ in continuous wave, $6 \mathrm{~J}$ ) or LPLT ( $\lambda 635 \mathrm{~nm}, 10 \mathrm{~mW}$ in continuous wave, $6 \mathrm{~J})$, with the plate cover left open during irradiation. At different time points after laser irradiation, cell viability and metabolism were measured with the ATPlite Luminescence Assay System (PerkinElmer, Waltham, MA) and the alamarBlue assay (Life Technologies Inc., Carlsbad, CA), according to the manufacturers' instructions. At the same time points, cells were incubated for 1 hour with $10 \mu \mathrm{mol} / \mathrm{L}$ bromodeoxyuridine (BrdU), followed by anti-BrdU immunostaining.

\section{Histology and Immunofluorescence}

For histopathological evaluation, 5- $\mu \mathrm{m}$ tissue sections were stained with hematoxylin and eosin. For immunofluorescence staining, tissue sections were stained with fluorescein isothiocyanate-labeled lectin (Lycopersicum esculentum; Vector Laboratories, Burlingame, CA), cyanine 3-labeled anti- $\alpha$-SMA antibodies (Sigma-Aldrich, St. Louis, MO), anti-BrdU (Abcam Inc., Cambridge, MA), or anti-NG2 antibodies (Chemicon International, Temecula, CA), followed by Alexa Fluor 555 secondary antibodies (Life Technologies Inc.). Image acquisition was performed with a confocal microscope (LSM510 META; Carl Zeiss, Jena, Germany) and an ImageXpress Micro high-content screening microscope (Molecular Devices, Sunnyvale, CA).

\section{Statistical Analysis}

Statistical analysis on both human and animal data was performed by SPSS software version 8.0 (SPSS Inc., Chicago, IL). A $P$ value $<0.05$ was considered statistically significant. Wilcoxon test was performed to obtain the significance of the differences in the healing parameters (VAS and CTC) on laser treatment, as well as in lesion size (erythema and ulceration) before and after laser therapy. A Student's $t$-test was performed to evaluate the mean duration of OM compared with literature data. McNemar's test was performed to evaluate the significance of the differences in subjective discomfort regression before and after laser therapy, as well as in the number of patients affected by swallowing, phonatory, and chewing pain. $\chi^{2}$ Test (exact Fisher's test) was performed to evaluate the influence of sex and age on OM regression. $\chi^{2}$ Test (Pearson's test) was used to correlate the regression of OM and type of neoplasia.

For animal data, two researchers independently performed histopathological examination. Raw data were transformed into a score index for each parameter, as detailed in Supplemental Table S2. Statistical significance of the differences in the histopathological scores among the time points within each group and among the groups within each time point was assessed by two-way analysis of variance for repeated measurements and Kruskal-Wallis and MannWhitney tests.

\section{Results}

\section{LPLT Improves OM Healing in CT-Treated Patients}

Baseline demographic and clinical characteristics of the patients are reported in Table 1. All patients underwent the same protocol of laser application and follow-up (Figure 1A). By using either CTC or VAS, laser therapy appeared effective in patients of all ages and of both sexes $(P<0.001$, respectively) (Figure 1B).

All patients tolerated LPLT, and none reported any major adverse event. Functional capacity was remarkably improved by LPLT because swallowing, phonatory, and chewing capacities were completely recovered in $100 \%$ of patients after treatment $(P<0.05)$ (Figure $1 \mathrm{C})$. In addition, the subjective perception of changes in seven different parameters (voice alteration, problems in swallowing, lips alteration, saliva alteration, tongue alteration, mucosae 
A
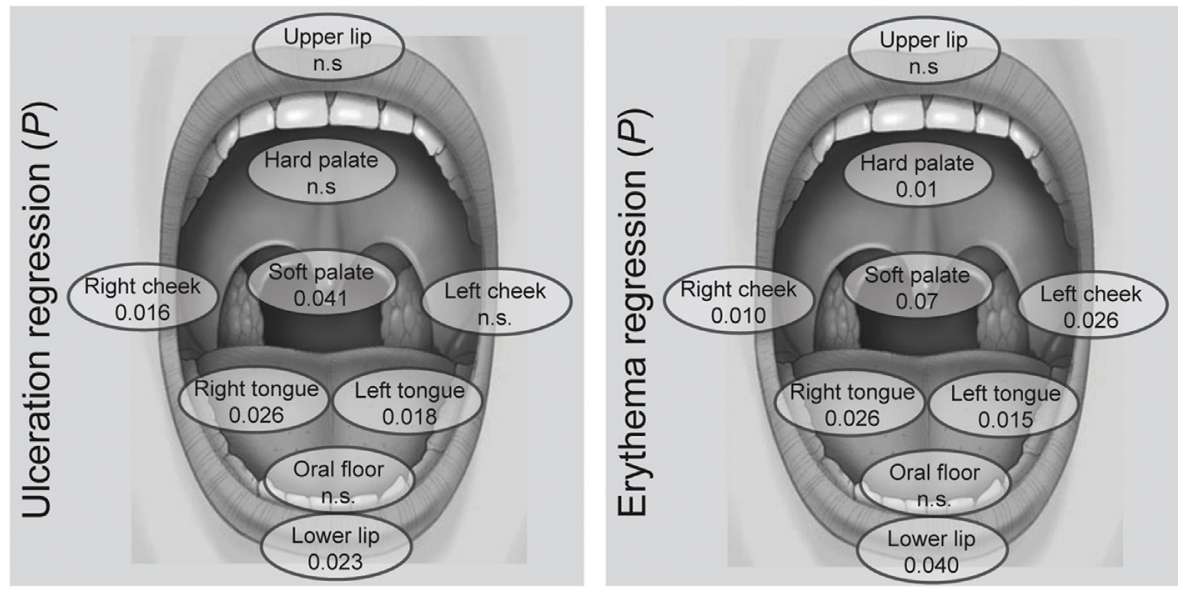

B

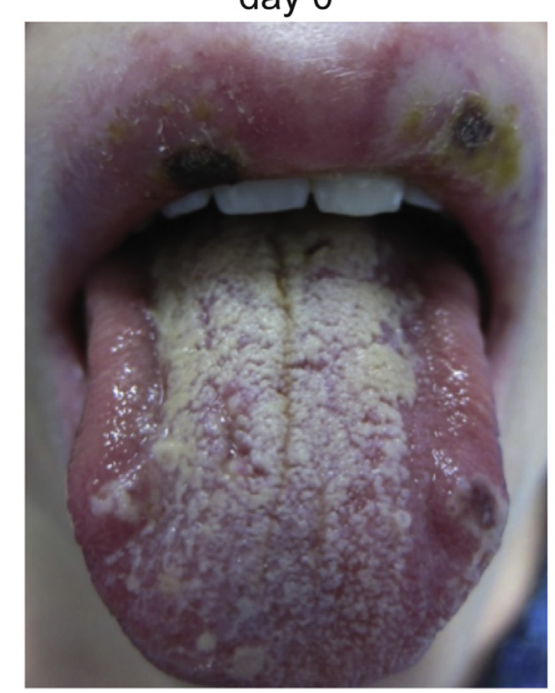

C

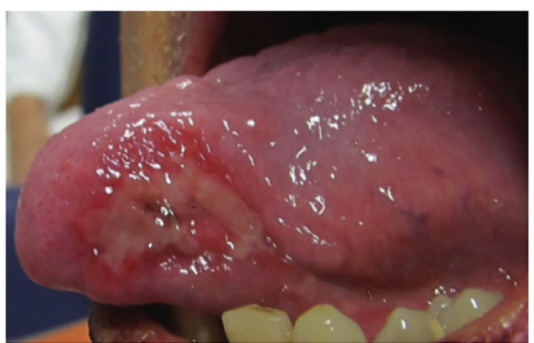

D

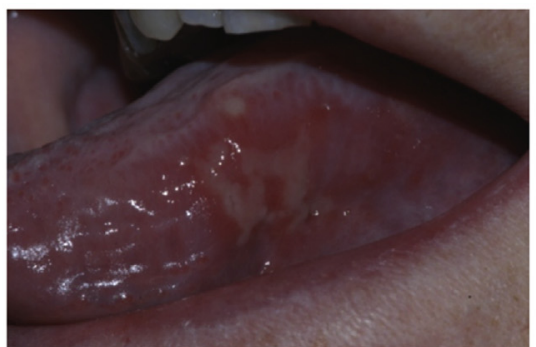

day 4
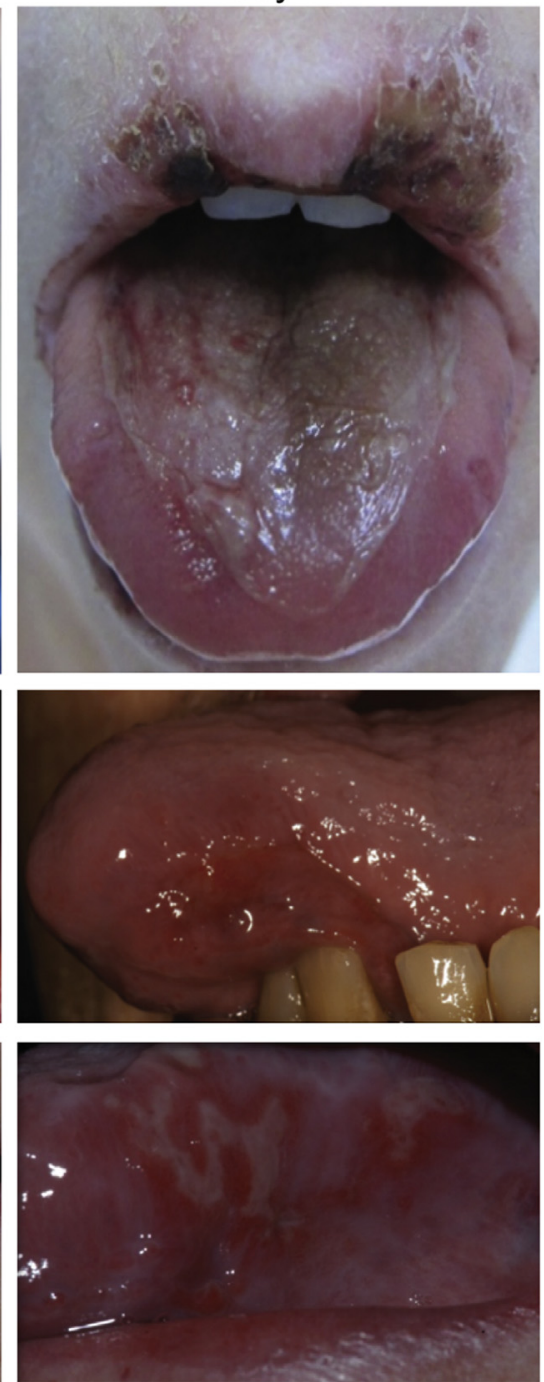

day 25
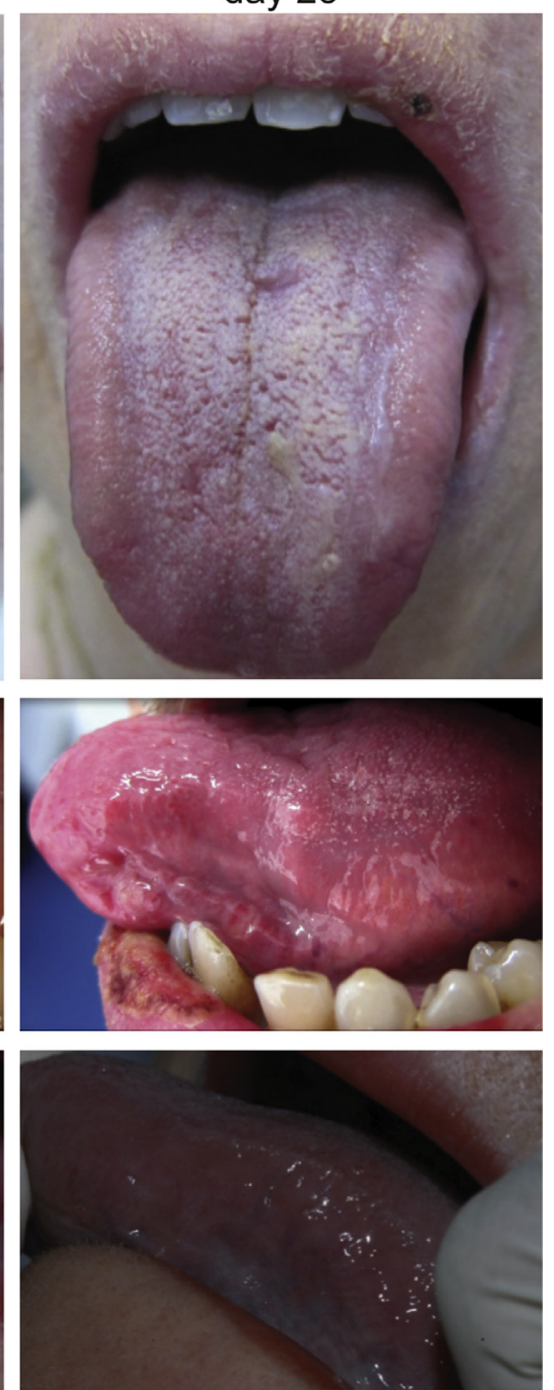

Figure 2 Clinical evaluation of OM severity after LPLT. A: Schematic representation of the different areas of the oral cavity examined before treatment and during follow-up. For each area, the $P$ value indicates the statistical significance of the differences observed in the severity of ulceration and erythema at day 0 and day 25, respectively. n.s., not significant. B-D: Representative images of three patients belonging to the three classes of neoplasia considered in our study, taken before treatment (day 0), immediately after treatment (day 4), and at the end of the follow-up period (day 25). B: Evolution of the 0M on the tongue of a patient affected by a chronic lymphocytic leukemia, treated with consecutive cycles of fludarabine and cyclophosphamide, alemtuzumab, highdose methylprednisolone, and rituximab. C: Evolution of the $0 \mathrm{M}$ on the tongue of a patient affected by a pharyngeal carcinoma, treated with cisplatin and 5 fluorouracil. D: Evolution of the $\mathrm{OM}$ on the tongue of a patient affected by a colorectal cancer, treated with carboplatin. 
alteration, and problems in performing a good oral care) were significantly decreased after LPLT $(P<0.05)$ (Figure 1D). This latter result appears of particular relevance, because proper oral hygiene is known to significantly reduce the incidence of $\mathrm{OM}^{18}$

Clinical evaluation with the use of CTC (Table 2) indicated that oral lesions completely healed 3 weeks after LPLT in $70 \%$ of patients, resulting in being totally asymptomatic. The remaining $30 \%$ of patients indicated a total remission of pain, despite lesions being still present, although less severe. Laser application was performed on different areas of the oral cavity; regardless of the area considered, LPLT was beneficial in improving lesion healing (Figure 2A) and subsequent pain remission. Representative images of the healing of oral lesions in three patients, who underwent $\mathrm{CT}$ for a chronic lymphocytic leukemia (class 1), a pharyngeal carcinoma (class 2), and a colon cancer (class 3), respectively, are shown in Figure 2, B-D. Patients affected by hematological neoplasias seemed to heal better than patients affected by solid or head and neck cancer $(P=0.05)$.

Approximately $15 \%$ of patients experienced some burning immediately after laser application, not associated with pain. No correlation was found between initial CTC score and healing of lesions, pain remission, or experience of burning after treatment. This might be partially explained because the clinical aspect of the lesions is not always directly proportional to the severity of pain (VAS) or the OM grade (CTC), and apparently mild lesions can be extremely debilitating and compromise oral function.

\section{Laser Therapy on a Mouse Model of CT-Induced OM: Study Design}

Because the harvesting of excisional biopsies from healing OMs after LPLT was not ethically acceptable, we established a mouse model of CT-induced OM to evaluate the structural changes induced by laser treatment to the treated tissues.

OM was induced on mice by 5-fluorouracil treatment, followed by topic application of acetic acid to the underside of the tongue. Animals were randomly assigned to two experimental groups, receiving LPLT and HPLT, respectively, and to a control group, which did not receive any laser treatment. All mice were then monitored daily and individually for survival, body weight, and clinical assessment of OM severity. At different time points, excisional biopsies of the tongue were obtained for histological analysis (Supplemental Table S3).

\section{LPLT and HPLT Improve Healing in a Mouse Model of CT-Induced OM}

The protective effects of laser therapy against CT-induced $\mathrm{OM}$ were first evaluated clinically. The CT regimen resulted in substantial $(>20 \%)$ body weight loss, followed by a slow recovery, which was almost complete by day 10 in all three experimental groups (Figure 3A). Clinical inspection of the oral cavity offered visual evidence of extensive mucosal damage and inflammation, with extensive ulceration, pseudomembranes, hemorrhage, and erythema, in all three experimental groups at day 4 after the induction of OM (criteria considered to score the severity of mucosal lesions are presented in Supplemental Table S2). Although the severity of most of the lesions progressively decreased in all of the groups, lesions in control mice did not heal completely, resulting in a significant disease burden still present at day 10, which was the final time point of the study (OM score, $1.9 \pm 0.08$ ) (Figure 3B). In contrast, both laser regimens, but most importantly HPLT, remarkably improved and accelerated the healing process, resulting in a lower OM score at the final time point $(\mathrm{OM}$ score, $1.6 \pm 0.06$ and $0.9 \pm 0.07$ for LPLT and HPLT, respectively) (Figure 3B). Histological assessment of the extent of tongue mucosal damage at day 4 found a marked reduction in mucosal thickness, with decreased corneal layer and basal layer epithelial cellularity, associated to extensive tongue erosions, deep inflammatory infiltration into the underlying submucosal tissue, and massive degeneration of the tissue architecture in all groups (Figure 3C). However, in control mice, the signs of inflammation and degeneration were still present and, in some cases, even increased at day 10. Laser therapy allowed a marked healing of the lesions, with a thinner inflammatory infiltrate (Figure 3, C and D), almost complete disappearance of ulcerations and degenerative vacuoles (Figure 3, C and E), associated to marked preservation of tongue muscular bundles in the LPLT and HPLT, respectively (Figure 3, C and F). To assess the effect of laser therapy on neo-angiogenesis, tissue sections from the different groups were double-stained with fluoresceinated lectin, to label endothelial and mononuclear inflammatory cells, and with anti- $\alpha$-SMA antibodies, to label SMCs. As evident from the whole sections and respective magnifications shown in Figure 4, A-F, although in the control group the inflammatory infiltrate, corresponding to the green area, appeared less intense but similarly extended at day 10 (Figure 4D) compared with day 4 (Figure 4A), both laser treatments resulted in a limited inflammation at day 10, essentially confined to the site of the healing ulcer (compare Figure 4E with Figure 4B and Figure 4F with Figure 4C). In addition, a marked number of $\alpha$-SMA ${ }^{+}$vessels, reminiscent of newly formed arterioles, appeared in the laser-treated tissues at day 10 (compare Figure 4, E and F, with Figure 4D), consistent with massive arteriogenesis occurring within the granulation tissue formed during the healing process. Because $\alpha$-SMA is similarly expressed by both SMCs and myofibroblasts, we simultaneously stained the HPLT-treated tongues with anti$\alpha$-SMA and anti-NG2 antibodies, to label vascular pericytes, and found a remarkable number of double-positive cells (Supplemental Figure S1A). In addition, we also performed HPLT on $\alpha$-SMA-RFP/Coll $\alpha 1$ (I)-EGFP mice. As shown in Supplemental Figure S1B, red fluorescence appears 
A

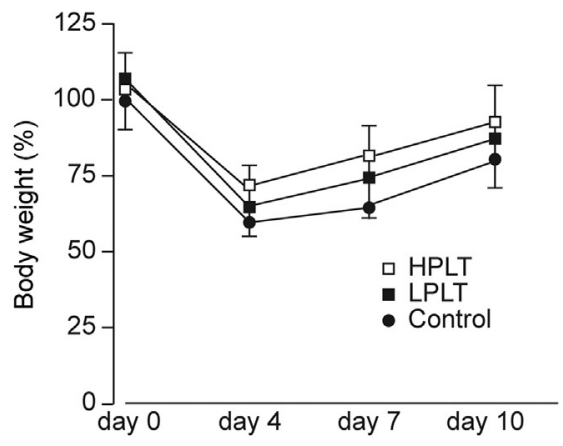

B

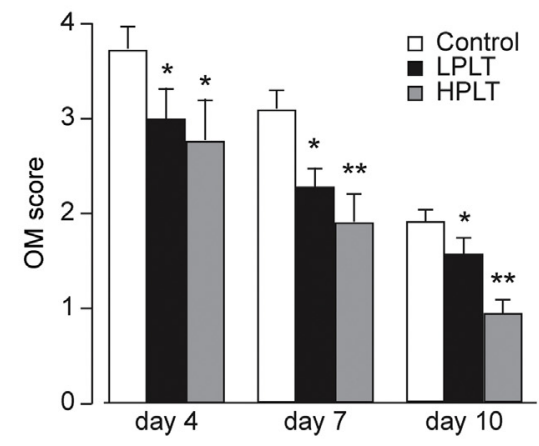

C
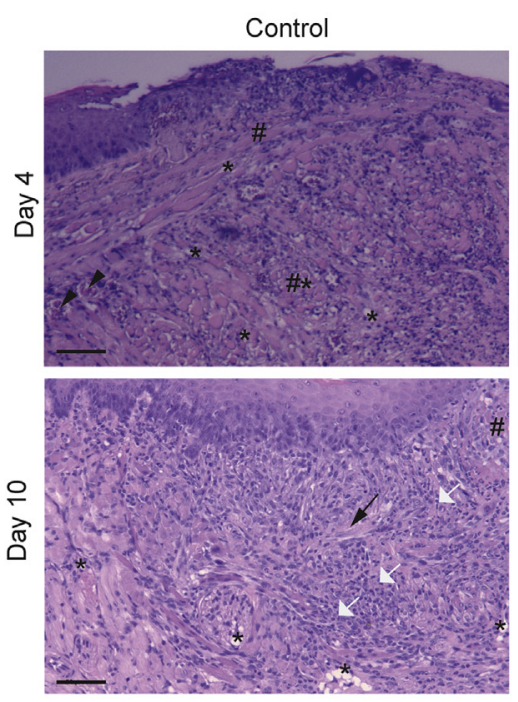
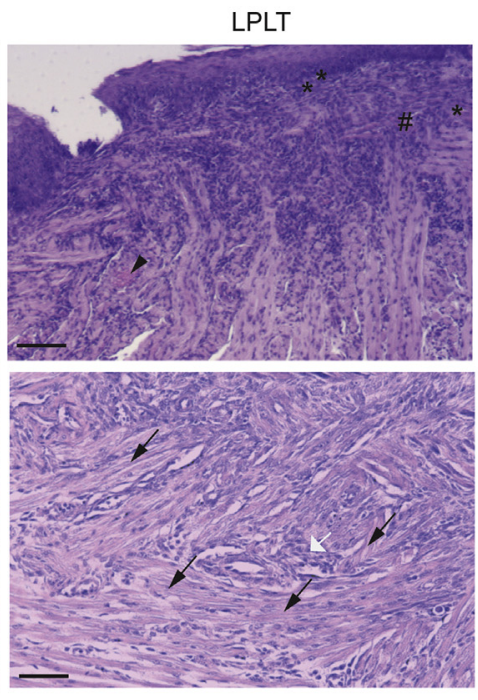

$\mathbf{E}$

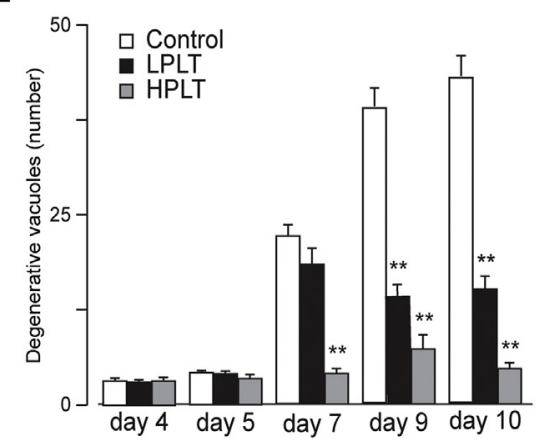

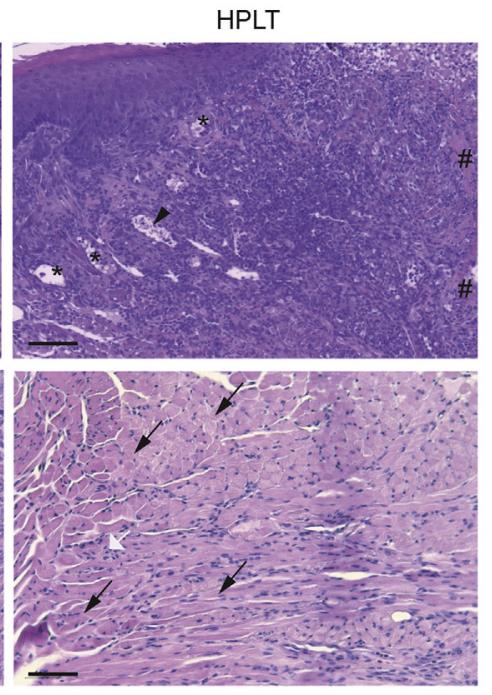

$\mathbf{F}$

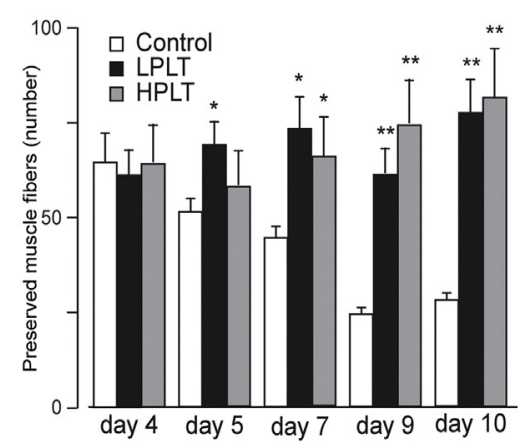

Figure 3 Effect of laser treatment on a mouse model of OM. A: Monitoring of body weight, expressed as a percentage of the body weight before the induction of OM. Data are expressed as means \pm SEM. B: Evaluation of the severity of OM according to a clinical score. Data are expressed as means \pm SEM. C: H\&E staining of sections of tongues with OM in the absence of treatment (left panels, control), after LPLT or HPLT at day 4 and day 10, respectively. Asterisks indicate degenerative vacuoles, pound signs indicate necrosis caseosa, arrowheads indicate hemorrhage, white arrows indicate lymphocytic infiltrates still present at day 10, and black arrows indicate preserved muscle fibers. Scale bar $=100 \mu \mathrm{m}$. D: Quantification of maximum deepness of the inflammatory response observed in the three experimental groups at the time points indicated. Data are expressed as means \pm SEM. E: Quantification of the number of degenerative vacuoles per microscopic field in the three experimental groups at the time points indicated. Data are expressed as means \pm SEM. F: Quantification of the number of intact muscle fibers per microscopic field in the three experimental groups at the time points indicated. Data are expressed as means \pm SEM. ${ }^{*} P<0.05,{ }^{* *} P<0.01$ versus control. Original magnification: $\times 20$ (E and $\left.\mathbf{F}\right)$.

specifically localized in vascular SMCs, whereas green fluorescence is detectable in isolated fibroblasts, and no double-positive cells are present in these sections. Overall, these results indicate that a major effect of laser therapy is the formation of a remarkable number of new arterial vessels. The quantification of the number of $\alpha$-SMA ${ }^{+}$arterioles observed in the different groups over time is provided in Figure $4 \mathrm{G}$. 

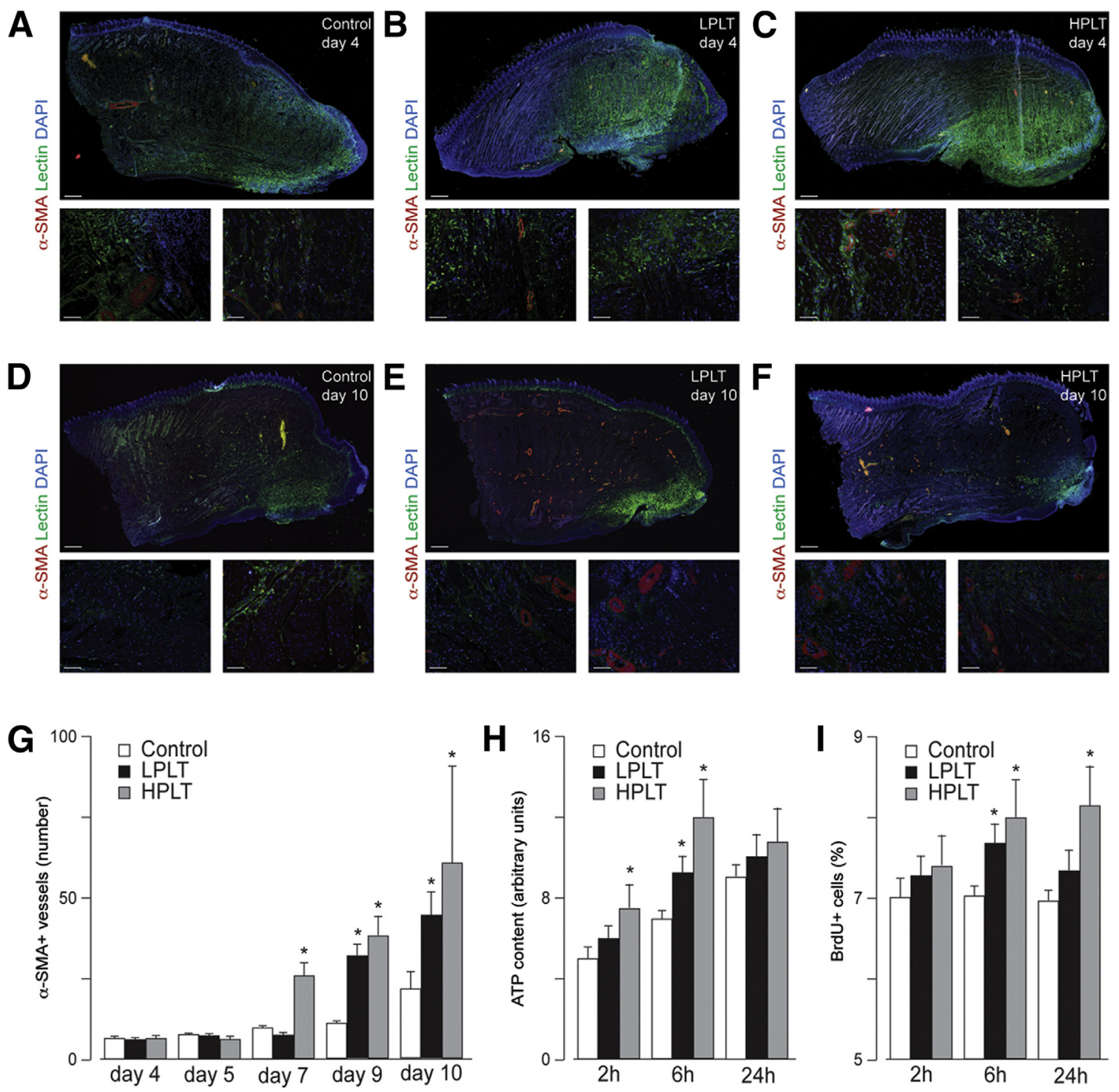

Figure 4 Effect of laser treatment on smooth muscle cell activation and arteriogenesis. A-F: Lectin and $\alpha$-SMA staining of tongues at day 4 after 0 M induction in the absence of treatment (A), after LPLT, (B) or HPLT (C) and at day 10 in the absence of treatment (D), after LPLT (E), or after HPLT (F). G-I: Quantification of the number of $\alpha$-SMA ${ }^{+}$arteries per microscopic field in tongue samples with OM (G), of ATP content in SMC's (H), and of BrdU ${ }^{+}$SMCs (I) in the absence of treatment (white columns) and after exposure to LPLT (black columns) or to HPLT (gray columns) at the time points indicated. Data are expressed as means \pm SEM. ${ }^{*} P<0.05$ versus control. Scale bars: $1 \mathrm{~mm}$ (whole tongue image, $\mathbf{A}-\mathbf{F}$ ); $100 \mu \mathrm{m}$ (magnifications below, A-F). Original magnification: $\times 10(\mathbf{A}-\mathbf{F})$.

\section{Laser Irradiation Improves Smooth Muscle Cell Metabolism and Proliferation}

To investigate whether the arteriogenic response elicited by laser treatment in vivo might stem from a direct effect of laser irradiation on vascular SMC metabolism and proliferation, we exposed cultured primary SMCs, derived from human coronary arteries, to $635 \mathrm{~nm}$ (LPLT) and $970 \mathrm{~nm}$ (HPLT) laser irradiation. At different time points, we assessed cell metabolic activity by measuring ATP intracellular content. We found that both laser regimens, but most effectively HPLT, significantly increased ATP content at 2 and 6 hours after irradiation (Figure $4 \mathrm{H}$ ). No significant changes in cell metabolic activity or viability were observed at later time points up to 5 days after irradiation (not shown), indicating absence of toxicity of laser treatment. To confirm a direct stimulation of laser treatment on smooth muscle metabolism, and possibly on their proliferation, we also performed alamarBlue assay, which measures the reducing power of living cells to convert the resazurin substrate into a fluorescent compound, thereby providing a quantification of cell metabolism and viability. We found a significant increase in the alamarBlue signal induced by HPLT (Supplemental Figure S1C). Finally, we administered BrdU to cultured SMCs, to count the number of proliferating cells as cells that had incorporated BrdU into their nuclei during DNA 
synthesis. Consistent with the previous results, we found that both laser regimens, but most effectively HPLT, promoted DNA synthesis, a prerequisite for cell proliferation, in vascular SMCs (Figure 4I).

\section{Discussion}

In this study we have evaluated and quantified the therapeutic effects of LPLT against CT-induced OM and its associated morbidities in a clinical setting, as well as in a mouse model of the disease. In addition, with the use of the same animal model we quantitatively compared the efficacy of LPLT with that of an innovative laser protocol, based on higher energy, defined as HPLT. Finally, we assessed the direct effect of the two different wavelengths on primary SMCs. This study is particularly innovative because i) for the first time it correlates clinical and experimental results on the efficacy of laser therapy against CT-induced OM; ii) it represents the first direct comparison of two laser regimens in vivo and in cell culture, thus paving the way for novel clinical experimentations with the use of HPLT; and iii) it provides clear-cut evidence of the capacity of laser therapy to induce the formation of new arterioles in vivo and to activate cultured SMCs.

Analysis of our results showed that therapeutic application of LPLT was effective in reducing the severity of OM and its associated pain, as well as in shortening the OM regression time, compared with the standard regression time, as indicated by the literature (from 21 to 24 days to 6 to 13 days with the use of either the VAS or the CTC scale). ${ }^{19}$

Similarly, most of the previous studies, ${ }^{20-22}$ including two recent meta-analyses, ${ }^{23,24}$ reported reduction on OM severity with the use of LPLT despite notable variations in study population, LPLT dosage, and outcomes for OM scoring. The last review of mucositis guidelines by the Mucositis Study Group of the Multinational Association of Supportive Care in Cancer/International Society of Oral Oncology ${ }^{25}$ recognized the following limits for clinical application of LPLT: requirement of expensive equipment and specialized training, notable interoperator variability, difficult design and implementation of clinical trials, and difficult comparison of the results of clinical trials. Nevertheless, accumulating evidence in support of LPLT suggests that, for centers able to support the necessary technology and training, LPLT should be used to attempt to reduce the incidence of $\mathrm{OM}$ and its associated pain in patients receiving high-dose CT or CT before hematopoietic stem cell transplantation. This represented a significant change from previous guidelines, in which the use of laser therapy was only a suggestion for these patients. In fact, LPLT is becoming the reference standard to reduce OM-associated pain and to improve quality of life in the interval between consequent CT cycles. By contrast, the potential of LPLT to reduce the severity, duration, and symptoms of CT-induced
OM has tremendous economic implications. The number of complications that can arise as a consequence of OM necessitates an increased cost of US \$2725 per person for each CT cycle in case of grade 1 to 2 OM and US $\$ 5565$ in case of grade 3 to $4 \mathrm{OM}$, and this surplus is further exacerbated in case of concomitant RT. ${ }^{26}$

Can LPLT be considered more effective than the numerous alternative therapeutic options that claim to prevent or reduce severity of OM?

Two recent, systematic reviews in the available literature, which aimed at defining clinical practice guidelines for the prevention and treatment of OM, concluded that moderateto-strong evidence exists in favor of laser therapy applied to the oropharyngeal area in patients with cancer who receive $\mathrm{CT}$ or RT in both the prevention of OM occurrence and in the reduction of the severity, pain, and duration of $\mathrm{OM}$ ulcers. ${ }^{24,27}$ Interestingly, as similarly observed in our study, the variety of different cancers and relative therapies involved in the trials examined did not seem to seriously interfere with the beneficial effects of laser therapy. ${ }^{24}$ The reviews also suggested that future studies should specifically examine the effect of altering laser parameters and, in particular, different wavelengths, as well as assessing the effect of phototherapy, in animal models of OM, focusing on elements in the pathobiological process of OM. ${ }^{27}$ In this study, we have taken up precisely these suggestions and have explored in a comparative manner the effects of two different laser wavelengths $(635 \mathrm{~nm}$ and $970 \mathrm{~nm})$ and energy power $(2.5 \mathrm{~mW}$ in continuous wave and $50 \%$ duty cycle at $5 \mathrm{~W}$ ) in an animal model of CT-induced OM. In particular, because the biostimulating and anti-inflammatory effects depend on the penetration of the beam and are affected by both surface scattering and water, melanin, and hemoglobin absorption, we hypothesized that HPLT could be more effective in concentrating higher energy on a more superficial mucosal layer. Our results clearly indicate that the two protocols are not redundant in terms of efficacy, because HPLT induced better healing and reduced inflammation than did LPLT. This is also in agreement with the notion that laser irradiation always exerts a thermal effect, which, in the long term, can result in architectural damage, interstitial edema, disarrayed muscle fibers, and cytoplasmic vacuolization. A limited thermal effect is likely to have occurred in the HPLT group, which was characterized by extraordinary tissue integrity, consistent with the shallower penetration of high wavelengths. These results prompt the use of HPLT as a novel, more effective strategy for the clinical management of CT-induced OM. Notably, although the design of a new clinical trial that includes assessing the efficacy of LPLT against a placebo group might be considered unethical in light of the clear benefit provided by LPLT for critical patients, possible comparison of the efficacy of the two laser regimens (LPLT versus HPLT) might embody a clinical study to be performed in the near future.

Besides assessing the efficacy of the different laser protocols, our study also provides important findings in terms 
of the mechanisms of action of the therapies proposed. One of the main limitations in understanding the molecular and cellular mechanisms responsible for the therapeutic activities of laser against CT-induced OM is the limited access to the treated tissues. These tissues cannot be biopsied directly from patients for ethical reasons. Thus, the availability of an animal model of the disease represents a valuable tool for analyzing the biological processes that occur within the treated tissues. Successful laser application in other diseases, such as osteoarthritis or tendinopathies, ${ }^{28}$ diabetic foot pain, ${ }^{29}$ and neck pain, ${ }^{30}$ have shown clearly that the potent anti-inflammatory and anti-edema effects of certain wavelengths essentially occur through acceleration of microcirculation and result in changes in capillary hydrostatic pressure and neoangiogenesis. ${ }^{31}$ In addition, a specific activity of LPLT in inducing fibroblast transformation into myofibroblasts has been proven in several clinical and experimental contexts. ${ }^{32}$ We believe that our results add an important piece of information by showing that both laser regimens, most importantly HPLT, promote the formation of new arterioles in the tissue to help heal the mucosal layer and act directly on SMCs to foster their metabolism and proliferation. This is particularly relevant because the newly formed vessels within the granulation tissue have to undergo structural and functional maturation to become impermeable and to sustain an increased blood flow. This is essential to accelerate the repair process. ${ }^{11,33}$

The improved effect of HPLT is in apparent contradiction with previous results that compare the effect of similar laser wavelengths ( 660 and $780 \mathrm{~nm}$ ) on a rat model of diabetic ulcer, in which the higher energy protocol displayed positive effects at early stages on the onset of inflammation but performed worse in terms of amount and quality of the granulation tissue at the end of the experimental period ( 3 weeks). ${ }^{34}$ This partial discrepancy might be reasonable because of the more complex microanatomy of the skin compared with the mucosa (the presence of a thick stratum corneum, a dense layer of dermal connective tissue, a hypodermal panniculus adiposus, and an additional layer of striated muscle, known as panniculus carnosum) and certainly justifies the requirement for a more penetrating, lower wavelength to obtain a biostimulatory effect on the rat skin.

In conclusion, although a standard treatment for CTrelated $\mathrm{OM}$ has not yet met with univocal agreement, mounting evidence points toward LPLT as the treatment of choice to foster OM healing and thereby improve quality of life and overall survival for these patients. Our results support the use of LPLT in all CT-treated patients who experience OM, regardless of their specific cancer and CT, and prompt the development of a clinical trial to compare the efficacy of LPLT and HPLT in humans.

\section{Acknowledgement}

We thank Suzanne Kerbavcic for excellent editorial assistance.

\section{Supplemental Data}

Supplemental material for this article can be found at http://dx.doi.org/10.1016/j.ajpath.2013.09.003.

\section{References}

1. Treister N, Sonis S: Mucositis: biology and management. Curr Opin Otolaryngol Head Neck Surg 2007, 15:123-129

2. Trotti A, Bellm LA, Epstein JB, Frame D, Fuchs HJ, Gwede CK, Komaroff E, Nalysnyk L, Zilberberg MD: Mucositis incidence, severity and associated outcomes in patients with head and neck cancer receiving radiotherapy with or without chemotherapy: a systematic literature review. Radiother Oncol 2003, 66:253-262

3. Ohrn KE, Sjoden PO, Wahlin YB, Elf M: Oral health and quality of life among patients with head and neck cancer or haematological malignancies. Support Care Cancer 2001, 9:528-538

4. Sonis ST, Oster G, Fuchs H, Bellm L, Bradford WZ, Edelsberg J, Hayden V, Eilers J, Epstein JB, LeVeque FG, Miller C, Peterson DE, Schubert MM, Spijkervet FK, Horowitz M: Oral mucositis and the clinical and economic outcomes of hematopoietic stem-cell transplantation. J Clin Oncol 2001, 19:2201-2205

5. Brizel DM, Wasserman TH, Henke M, Strnad V, Rudat V, Monnier A, Eschwege F, Zhang J, Russell L, Oster W, Sauer R: Phase III randomized trial of amifostine as a radioprotector in head and neck cancer. J Clin Oncol 2000, 18:3339-3345

6. Coghlin Dickson TM, Wong RM, offrin RS, Shizuru JA, Johnston LJ, $\mathrm{Hu}$ WW, Blume KG, Stockerl-Goldstein KE: Effect of oral glutamine supplementation during bone marrow transplantation. JPEN J Parenter Enteral Nutr 2000, 24:61-66

7. Culy CR, Spencer CM: Amifostine: an update on its clinical status as a cytoprotectant in patients with cancer receiving chemotherapy or radiotherapy and its potential therapeutic application in myelodysplastic syndrome. Drugs 2001, 61:641-684

8. Peterson DE: New strategies for management of oral mucositis in cancer patients. J Support Oncol 2006, 4:9-13

9. Lubart R, Eichler M, Lavi R, Friedman H, Shainberg A: Low-energy laser irradiation promotes cellular redox activity. Photomed Laser Surg 2005, 23:3-9

10. Gao X, Xing D: Molecular mechanisms of cell proliferation induced by low power laser irradiation. J Biomed Sci 2009, 16:4

11. Lins RD, Dantas EM, Lucena KC, Catao MH, Granville-Garcia AF, Carvalho Neto LG: Biostimulation effects of low-power laser in the repair process. An Bras Dermatol 2010, 85:849-855

12. Feng J, Zhang Y, Xing D: Low-power laser irradiation (LPLI) promotes VEGF expression and vascular endothelial cell proliferation through the activation of ERK/Sp1 pathway. Cell Signal 2012, 24: 1116-1125

13. Peplow PV, Chung TY, Baxter GD: Laser photobiomodulation of proliferation of cells in culture: a review of human and animal studies. Photomed Laser Surg 2010, 28(suppl 1):S3-S40

14. Worthington HV, Clarkson JE, Bryan G, Furness S, Glenny AM, Littlewood A, McCabe MG, Meyer S, Khalid T: Interventions for preventing oral mucositis for patients with cancer receiving treatment. Cochrane Database Syst Rev 2011, (4):CD000978

15. Gobbo M, Ottaviani G, Mustacchi G, Di Lenarda R, Biasotto M Acneiform rash due to epidermal growth factor receptor inhibitors: high-level laser therapy as an innovative approach. Lasers Med Sci 2012, 27:1085-1090

16. World Health Organization: Common Toxicity Criteria. Geneva, Switzerland, World Health Organization, 1976

17. Magness ST, Bataller R, Yang L, Brenner DA: A dual reporter gene transgenic mouse demonstrates heterogeneity in hepatic fibrogenic cell populations. Hepatology 2004, 40:1151-1159 
18. Rosenthal DI, Trotti A: Strategies for managing radiation-induced mucositis in head and neck cancer. Semin Radiat Oncol 2009, 19:29-34

19. Scully C, Sonis S, Diz PD: Oral mucositis. Oral Dis 2006, 12:229-241

20. Gautam AP, Fernandes DJ, Vidyasagar MS, Maiya AG, Vadhiraja BM: Low level laser therapy for concurrent chemoradiotherapy induced oral mucositis in head and neck cancer patients - a triple blinded randomized controlled trial. Radiother Oncol 2012, 104:349-354

21. Nes AG, Posso MB: Patients with moderate chemotherapy-induced mucositis: pain therapy using low intensity lasers. Int Nurs Rev 2005, 52:68-72

22. Schubert MM, Eduardo FP, Guthrie KA, Franquin JC, Bensadoun RJ, Migliorati CA, Lloid CM, Eduardo CP, Walter NF, Marques MM, Hamdi M: A phase III randomized double-blind placebo-controlled clinical trial to determine the efficacy of low level laser therapy for the prevention of oral mucositis in patients undergoing hematopoietic cell transplantation. Support Care Cancer 2007, 15:1145-1154

23. Bensadoun RJ, Nair RG: Low-level laser therapy in the prevention and treatment of cancer therapy-induced mucositis: 2012 state of the art based on literature review and meta-analysis. Curr Opin Oncol 2012, $24: 363-370$

24. Bjordal JM, Bensadoun RJ, Tuner J, Frigo L, Gjerde K, LopesMartins RA: A systematic review with meta-analysis of the effect of low-level laser therapy (LLLT) in cancer therapy-induced oral mucositis. Support Care Cancer 2011, 19:1069-1077

25. McGuire DB, Johnson J, Migliorati C: Promulgation of guidelines for mucositis management: educating health care professionals and patients. Support Care Cancer 2006, 14:548-557

26. Elting LS, Cooksley C, Chambers M, Cantor SB, Manzullo E, Rubenstein EB: The burdens of cancer therapy. Clinical and economic outcomes of chemotherapy-induced mucositis. Cancer 2003, 98: $1531-1539$

27. Migliorati C, Hewson I, Lalla RV, Antunes HS, Estilo CL, Hodgson B, Lopes NN, Schubert MM, Bowen J, Elad S; For the Mucositis Study Group of the Multinational Association of Supportive Care in
Cancer/International Society of Oral Oncology: Systematic review of laser and other light therapy for the management of oral mucositis in cancer patients. Support Care Cancer 2013, 21:333-341

28. Bjordal JM, Lopes-Martins RA, Joensen J, Couppe C, Ljunggren AE, Stergioulas A, Johnson MI: A systematic review with procedural assessments and meta-analysis of low level laser therapy in lateral elbow tendinopathy (tennis elbow). BMC Musculoskelet Disord 2008, 9:75

29. Kaviani A, Djavid GE, Ataie-Fashtami L, Fateh M, Ghodsi M, Salami M, Zand N, Kashef N, Larijani B: A randomized clinical trial on the effect of low-level laser therapy on chronic diabetic foot wound healing: a preliminary report. Photomed Laser Surg 2011, 29: 109-114

30. Chow RT, Johnson MI, Lopes-Martins RA, Bjordal JM: Efficacy of low-level laser therapy in the management of neck pain: a systematic review and meta-analysis of randomised placebo or active-treatment controlled trials. Lancet 2009, 374:1897-1908

31. Schindl A, Heinze G, Schindl M, Pernerstorfer-Schon H, Schindl L: Systemic effects of low-intensity laser irradiation on skin microcirculation in patients with diabetic microangiopathy. Microvasc Res 2002, 64:240-246

32. Ribeiro MA, Albuquerque RL Jr., Ramalho LM, Pinheiro AL, Bonjardim LR, Da Cunha SS: Immunohistochemical assessment of myofibroblasts and lymphoid cells during wound healing in rats subjected to laser photobiomodulation at $660 \mathrm{~nm}$. Photomed Laser Surg 2009, 27:49-55

33. Zacchigna S, Pattarini L, Zentilin L, Moimas S, Carrer A, Sinigaglia M, Arsic N, Tafuro S, Sinagra G, Giacca M: Bone marrow cells recruited through the neuropilin-1 receptor promote arterial formation at the sites of adult neoangiogenesis in mice. J Clin Invest 2008, 118:2062-2075

34. Meireles GC, Santos JN, Chagas PO, Moura AP, Pinheiro AL: Effectiveness of laser photobiomodulation at 660 or 780 nanometers on the repair of third-degree burns in diabetic rats. Photomed Laser Surg 2008, 26:47-54 